\title{
A NOTE ON COMPACT GROUPS
}

\author{
TA-SUN WU
}

\begin{abstract}
We show that the product of certain subsets in a compact connected topological group is the group itself.
\end{abstract}

Let $G$ be a connected topological group. It is weil known that $G$ is generated by any neighborhood $V$ of the identity 1 of $G$, i.e. $G=\cup_{n=1}^{\infty} V^{n}$. If $G$ is also compact, then $G=V^{k}$ for some $k$. It is natural to ask whether the above statement is true if we replace the neighborhood of 1 by some other types of subsets of $G$. The purpose of this note is to show one such possibility. Precisely, we prove:

Proposition. Let $G$ be a compact connected Hausdorff topological group and $\mu$ the (normalized) Haar measure on $G$. Let $A_{1}, A_{2}, \ldots$ be a sequence of Borel measurable sets in $G$ such that $\inf \mu\left(A_{i}\right)>0$. Then $G=A_{1} A_{2} \cdots A_{n}$ for some $n$.

First, we need

Lemma. Let $A$ and $B$ be Borel subsets in $G$ with positive measure. Then $A B$ has nonvoid interior.

Proof. This is a special case of a well-known general result. For a proof, see [3. (20.17), p. 296].

ProOF (OF THE PROPOSITION). We may suppose by the Lemma that $A_{1}$ is an open subset. There are elements $a_{1}, a_{2}, \ldots$ in $G$ such that $1 \in A_{1} a_{1}$, and $1 \in a_{n-1}^{-1} A_{n} a_{n}$, for $n>1$. Let $B_{1}=A_{1} a_{1}, B_{n}=a_{n-1}^{-1} A_{n} a_{n}$. Then $\mu\left(B_{n}\right)=\mu\left(A_{n}\right)$. Since $A_{1} A_{2} \cdots A_{n}$ $=B_{1} B_{2} \cdots B_{n} a_{n}^{-1}$, it suffices to prove the Proposition for the sequence $B_{1}, B_{2} \ldots$ of Borel sets containing the identity element.

Let $S=\left\{x \in G \mid x \in B_{i}\right.$ for infinitely many indices $\}$. Then $S=\cap_{k=1}^{\infty} \cup_{i=k}^{\infty} B_{i}$. We note that $l \in S$ and $\mu(S) \geqslant \inf \mu\left(B_{i}\right)$. Let $S^{*}$ be the semigroup generated by $S$, i.e. $S^{*}=\cup_{n=1}^{\infty} S^{n}$. Then $1 \in S \subset S^{*}$. Because $S$ has positive measure, by the Lemma we know that $S^{*}$ has nonvoid interior $W$. Now we shall prove that 1 is in the interior $W$ of $S^{*}$. Let $x$ be any element in $W$. Since $G$ is compact, the closure of the semigroup generated by $x^{-1}$ is a compact subgroup; in particular, it contains 1 . Thus $x^{-n}$ is in the neighborhood $x^{-1} W$ of 1 for some $n \geqslant 1$. Then $x^{1-n} \in W$, and $1=x^{n-1} \cdot x^{1-n} \in x^{n-1} W \subset W^{n}=W$. In other words, $S^{*}$ is a neighborhood of 1 . Since $G$ is connected we conclude that $G=S^{*}$ (cf. the beginning of this note). Finally, since $S^{*}$ is generated by the elements which appear infinitely often in the

Received by the editors September 1, 1982 and, in revised form, January 17, 1983.

1980 Mathematics Subject Classification. Primary 22C05, 22 D99. 
sequence $B_{1}, B_{2}, \ldots, S^{*} \subseteq \cup_{n=1}^{\infty} B_{1} B_{2} \cdots B_{n}$. Using the fact that $G=S^{*}$ is compact and each $B_{1} B_{2} \cdots B_{n}$ is open, we conclude that $G=S^{*}=B_{1} B_{2} \cdots B_{k}$ for some $k$, and the proof is complete.

REMARK. Observe that, given a sequence of open neighborhoods $V_{1} V_{2}, \ldots$ of 1 , it is not always true that $G=V_{1} V_{2} \cdots V_{k}$ for some $k$. Simple examples such as taking small intervals in the circle illustrate this fact.

For earlier works in this direction, but on abelian locally compact groups, we refer to $[1,2,5]$ and references therein. This note shows certain kinds of ergodicity of subsets in compact groups. For applications of this kind. we refer to [4].

\section{REFERENCES}

1. A. Beck, H. H. Corson and A. B. Simon, The interior points of the product of two subsets of a locally. compact group, Proc. Amer. Math. Soc. 9 (1958), 648-652.

2. A. Beck, A note on semi-groups in a locally compact group, Proc. Amer. Math. Soc. 11 (1960), 992-993.

3. E. Hewitt and K. Ross, Abstract harmonic analysis, Vol. 1, Springer, Berlin and New York, 1962.

4. H. Keynes and D. Newton, Lifting in non-abelian ( $G, 7)$-extensions, Lecture Notes in Math., vol. 668 , Springer-Verlag, Berlin and New York, 1977.

5. M. Kneser, Summenmengen in lokalkompakten abelschen Gruppen, Math. Z. 66 (1956), 88-110.

Department of Mathematics, Case Western Reserve University, Cleveland, Ohio 44106 